\title{
Virtual Reef: A Visualisation Framework for Marine Simulation Models
}

\author{
Stephen Jeffrey \\ Advanced Computational Modelling Centre, \\ University of Queensland, St. Lucia, 4072, Australia \\ sjj@maths .uq.edu. au \\ http://www. acmc.uq.edu.au
}

\begin{abstract}
A real-time application has been developed to enable immersive stereoscopic visualisation of the results of marine simulation models. Static datasets from external applications or dynamic output from realtime models may be interactively examined in a simulated underwater environment. The current work describes how the visualisation framework has been coupled to a morphogenetic model of coral growth and a biomechanical model of a fish. Coral structures have been modelled using the techniques developed by Kaandorp, and artificial fish have been constructed using methods adapted from the work of Tu.
\end{abstract}

\section{Introduction}

Mathematical models of complex systems are rarely complete; researchers are usually engaged in an iterative process of model refinement and output analysis. The technique used for assessing and/or comprehending model outputs usually depends on the nature of the simulation and also the size and complexity of the data. Visual techniques can be particularly useful, especially for three-dimensional datasets, as the human perception system is well adapted to performing graphical tasks such as pattern recognition. Graphical tools are therefore well suited to analysing outputs from environmental models given the spatio-temporal characteristics of many environmental processes.

The purpose of this paper is to describe the construction of a "virtual reef" that has been designed to facilitate the development of marine simulation models by using immersive visualisation. The graphical framework consists of an underwater scene and a range of navigation modes and visual effects. Datasets can be displayed either by first converting the data to a standard graphics format, or alternatively, the user may write their own $\mathrm{C} / \mathrm{C}++$ function for loading and rendering the data as desired. The conceptual layout of the framework is shown in Fig. 1.

Preliminary models of coral growth and fish movement have been implemented to demonstrate how model outputs may be examined in the graphical 


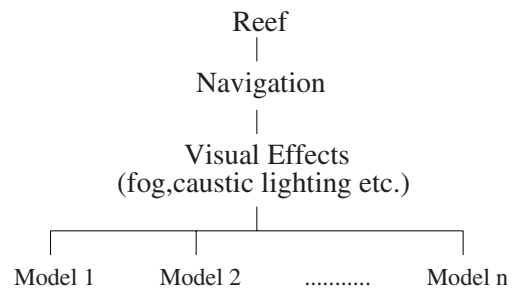

Fig. 1. Structure of the "virtual reef" application

environment. The coral and fish models are described in Sections 2 and 3, respectively, followed by implementation issues in Section 4. Concluding remarks are presented in Section 5 .

\section{Coral Growth Model}

A coral growth model based upon the work of Kaandorp (See [1] and references therein) has been used. The model attempts to simulate the radiate accretive growth process exhibited by many corals, sponges and algae. A longitudinal cross section through a colony of Montastrea annularis (Fig. 2) demonstrates

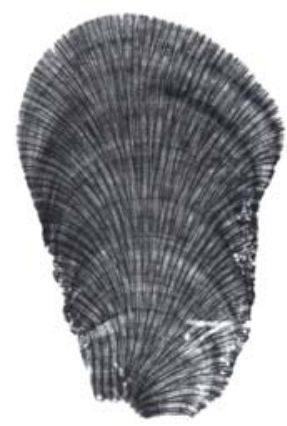

Fig. 2. Longitudinal cross section through a colony of Montastrea annularis. Photograph taken from [2]

the process: growth occurs in layers whereby a new layer is added to the existing structure. The growth is radial in nature due to the process's dependence on light and/or nutrients for growth. Growth is a maximum at the centre of the growth axis where the surface area (through which light and/or nutrients are absorbed) is a maximum and progressively decreases towards the side of the colony as the effective absorption area decreases. The two-dimensional growth model is shown in Fig. 3 where the surface elements are the tangential edges along the new 


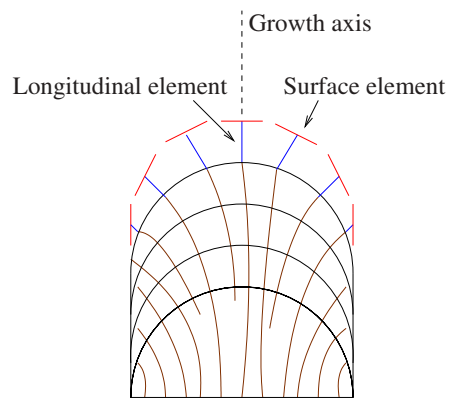

Fig. 3. Diagram shows the radiate accretive growth process. The lowest arc represents the initial state and the higher arcs represent successive growth layers. Diagram reproduced from [2]

outer surface, and the longitudinal elements show the growth between surface elements in successive layers. The basic growth model is given by:

$$
l=s \cdot \sin (\alpha), 0 \leq \alpha \leq \pi
$$

where $l$ is the distance grown, $s$ is the maximum growth and $\alpha$ is the angle between the surface tangent (at the point of interest) and the growth axis. Additional rules are progressively added to refine the model and are summarised in Table 1.

A three-dimensional model for radiate accretive growth can be constructed by a relatively straightforward extension of the two-dimensional model. In the $3 \mathrm{D}$ case, the 2D surface elements are replaced by triangles where each vertex represents the location of an individual polyp. The triangular structure is motivated by the fact that corallites commonly have a pentagonal and hexagonal arrangement which can be conveniently tessellated using triangles. The simulation starts with an initiator obtained by constructing a triangulated approximation to a sphere. Each vertex on the surface represents an individual polyp and is grown using a set of rules which are essentially $3 \mathrm{D}$ analogs of the $2 \mathrm{D}$ rules in Table 1. The early stages of the growth process are illustrated in Fig. 4. The structure on the left is the initiator which seeds the simulation. The middle structure is obtained after one growth iteration and it can be seen that one triangle has been subdivided into four smaller triangles. The subdivision is a result of the Insertion rule and it represents the emergence of new polyps. The structure on the right shows the colony branching. The structure has widened due to environmental factors and the effect is implemented by the Widening rule. The colony may widen if, for example, there is a predominant current resulting in the increased transportation of nutrients in a specific direction. Growth (for a heterotrophic species) will accelerate in the direction normal to the current. When the structure has widened sufficiently, growth may cease in the central area and two or more branches may emerge. The branches appear due to increased growth in regions with a relatively high surface area, through which light and/or nutrients are absorbed. This effect is implemented by one or more of the Contact, Light 
Table 1. Rules used in the model describing two-dimensional radiate accretive growth

\begin{tabular}{|c|c|}
\hline Rule & Description \\
\hline Growth & Distance grown (Eqn. 1) \\
\hline Continuity & $\begin{array}{l}\text { Adjacent surface elements form a smooth surface } \\
\text { (nearby polyps grow at similar rates) }\end{array}$ \\
\hline Insertion & $\begin{array}{l}\text { Insert a new surface element between adjacent surface elements that } \\
\text { are sufficiently far apart } \\
\text { (emergence of a new polyp) }\end{array}$ \\
\hline Inhibition & $\begin{array}{l}\text { Threshold below which growth is truncated } \\
\text { (polyp activity ceases) }\end{array}$ \\
\hline Widening & $\begin{array}{l}\text { Growth is accelerated on the side of the structure. This rule may } \\
\text { be used in basic models to approximate the effect of the Light and } \\
\text { Concentration rules } \\
\text { (all polyps within a region grow at a similar rate) }\end{array}$ \\
\hline Association & $\begin{array}{l}\text { Associate each surface element with a growth axis. A growth axis is } \\
\text { defined for each region of surface elements that are actively growing } \\
\text { (emergence of a new branch) }\end{array}$ \\
\hline Deletion & $\begin{array}{l}\text { Remove a surface element when adjacent surface elements are grow- } \\
\text { ing toward each other and are sufficiently close } \\
\text { (polyp growth suppressed to avoid collisions) }\end{array}$ \\
\hline Deregulation & $\begin{array}{l}\text { Random component incorporated into the Growth rule } \\
\text { (polyp growth is disturbed by surface irregularities or turbulence in } \\
\text { the environment) }\end{array}$ \\
\hline Contact & $\begin{array}{l}\text { Growth is made a function of the local radius of curvature } \\
\text { (polyp growth is influenced by the surface area through which it } \\
\text { can absorb light \&/or nutrients) }\end{array}$ \\
\hline Light & $\begin{array}{l}\text { Growth is made a function of the angle between the light source, } \\
\text { the point of interest (on the surface) and the surface normal at that } \\
\text { point. The light model provides a simple estimate of the available } \\
\text { light. A realistic model is not used as it would be too expensive to } \\
\text { compute } \\
\text { (polyp growth is dependent upon light) }\end{array}$ \\
\hline Concentration & $\begin{array}{l}\text { Growth is made a function of the local nutrient concentration, com- } \\
\text { puted using the discrete Laplace equation } \\
\text { (polyp growth is dependent upon nutrient concentration) }\end{array}$ \\
\hline
\end{tabular}



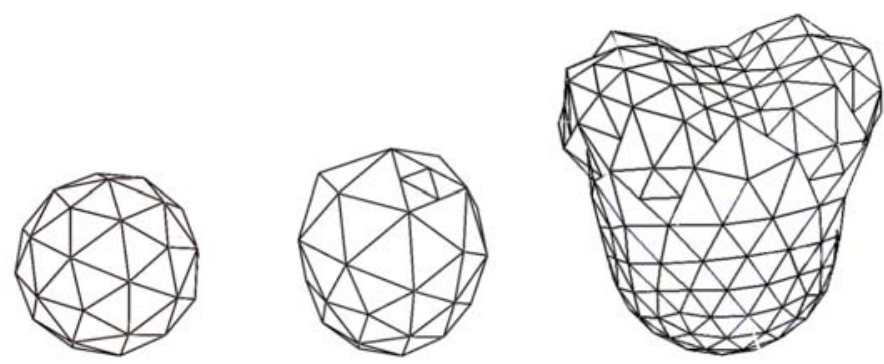

Fig. 4. Simulated radiate accretive growth in 3D. The structure on the left is the initiator, the middle structure is the form after one growth iteration and the structure on the right shows the colony branching. For further details, see the text

and Concentration rules. The fertile regions remaining after the branching then continue to grow along separate growth axes. Growth along these axes will be determined by each polyp's relationship with its new axis (as opposed to the axis prior to branching) and other environmental factors. Polyps are assigned to new growth axes by the Association rule.

The three-dimensional growth algorithm is built up from a set of rules similar to those used in the two-dimensional case. Further details on the growth algorithm may be found in [2].

\section{$3 \quad$ Fish Model}

Artificial fish have been constructed using a technique adapted from Tu's work [4]. The fish model consists of 3 distinct components: perception, behaviour and locomotion. The perception module is used to determine which objects are in the fish's field of view. An advanced recognition algorithm is not required as a list of all objects in the scene is known and a simple line of sight algorithm is used. The behaviour module takes input from the perception module and determines what action to take. A list of possible actions is constructed based upon the fish's knowledge of the location of other fish and its current desires and habits. Desires include the fish's intention to eat when hungry, flee from predators or school with other fish when appropriate. The candidate actions are ranked in order of priority and are executed by the locomotion module. Actions are prioritised to determine the most appropriate course to take in the current situation. For example, if the perception module has identified a nearby predator and the fish is hungry, the action selection mechanism should instruct the fish to flee. The desire to forage for food should be secondary to the fish's survival and executed after the fish has evaded the predator. The locomotion module consists of a mass-spring system representing a simplified model of the fish's muscle structure, and a set of motor controllers for activating the "muscles". Each motor controller is responsible for controlling the muscle actions required to implement a specific behaviour e.g., swimming, gliding, turning etc. 
The mass-spring system consists of 91 damped visco-elastic units arranged as in Fig. 5. The fish dynamics are simulated by numerically propagating the equations of motion over time. The governing equations are expressed as a set of coupled non-linear second order differential equations and solved numerically using a 4,5 Runge-Kutta algorithm with an adaptive step size [5]. In each time

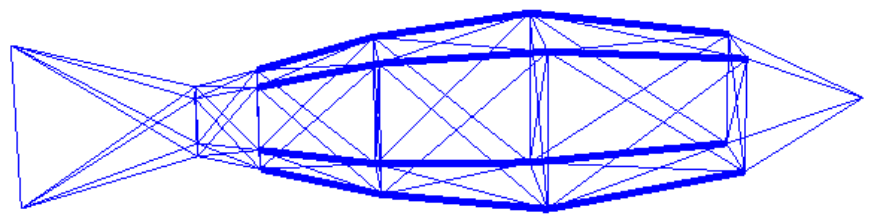

Fig. 5. The fish's internal structure. Each line represents a damped visco-elastic unit; muscles are shown as thick lines

step the motor controllers update the desired muscle lengths which then exert a force on the connecting nodes. The equations of motion are solved and the position of each node is updated.

The fish can be brought into a desired conformation by using an appropriate sequence of muscle actions. For example, a periodic relaxation and contraction of the tail muscles (muscles on either side are out of phase by $180^{\circ}$ ) make the tail oscillate [6]. The induced hydrodynamic forces propel the fish forward, thus simulating a swimming motion.

The fish body is made by mounting an external frame around the internal mass-spring system (Fig. 6). The offset between vertices in the external frame

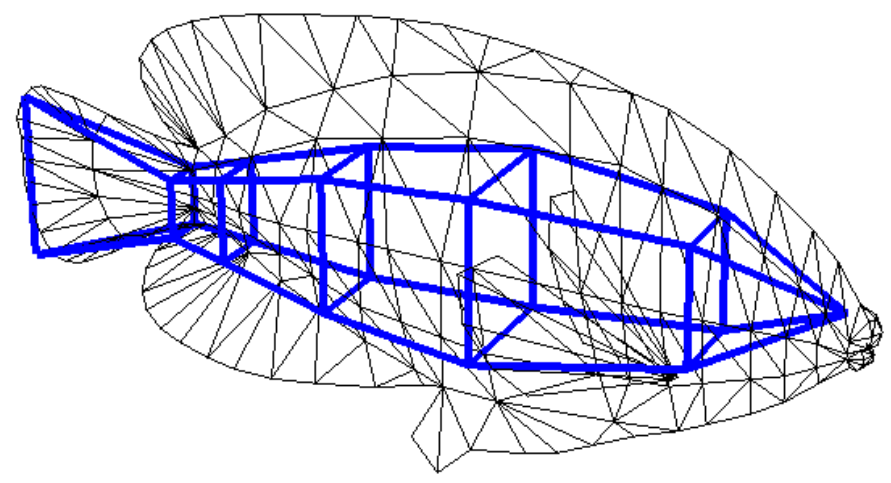

Fig. 6. The fish's body shape is given by mounting an external mesh relative to the internal frame 
and internal nodes is maintained as the internal frame moves. The offset ensures the fish's external shape deforms to match the internal structure.

Work on the perception and behaviour modules is not yet complete; the fish are currently controlled using key strokes i.e., the user sends commands directly to the locomotion module.

\section{Implementation Details}

The virtual reef has been designed for immersive stereoscopic visualisation. Its target machine is an SGI Onyx 3000, projecting three channels onto a curved screen $2.5 \mathrm{~m}$ high with a $150^{\circ}$ field of view. The software has been written in $\mathrm{C}++$ and uses OpenGL Performer [7]. Visual effects such as fog and caustic lighting have been used to enhance the immersive experience. Caustic lighting effects improve the realism of the underwater scene and are applied using texture-based colour modulation [8]. The SGI hardware does not support multi-texturing so a multi-pass rendering algorithm is used to implement the caustic effects. Fog is used to simulate a turbid environment but cannot be applied in the usual manner as it does not work correctly in the multi-pass environment. Fog is applied to each pixel using a one-dimensional texture map, where the texture coordinate is the normalised eye-distance. The eye-distance to each pixel is computed by reading the depth buffer and reversing the perspective scaling.

The application has been demonstrated by using it to display colonies of coral generated by a morphogenetic growth model. Texture mapping has been used to make the coral visually appealing but its application presents some interesting challenges. The growth algorithm was described in Section 2 where it was noted that vertices in the triangulated surface represent the location of an individual polyp. To reflect this one-to-one correspondence between vertices and polyps, a texture map of a single corallite can be centred on each vertex comprising the surface. The method can be used on structures generated by the coral growth algorithm, but is unsuitable in practice as the original structures undergo further processing. To reduce the graphics load, polygon reduction and level-of-detail techniques are used, thereby removing the correspondence between vertices and polyps. Since vertices in the processed structures no longer have any biological significance, there is no need to incur the graphics overhead associated with centering a texture map on each individual vertex. In practice textures are applied using automatic texture coordinate generation based upon distances from multiple planes.

The application can simultaneously display model outputs from a number of diverse models as the graphics are independent of the underlying simulation algorithms. The examples presented in Sections 2 and 3 demonstrate the separation between models and graphics. The coral simulation is an external application that generates coral structures which are then added to the underwater scene at run time. The fish simulation is compiled into a dynamic library and linked at run time. Since both algorithms are independent of the visualisation application, alternate simulation models can be easily substituted. 


\section{Conclusion}

A visualisation framework has been described which may be used to analyse the outputs of marine simulation models. Example applications have been developed and demonstrate how the graphical environment can assist the development of models by enabling researchers to interactively examine their results. In addition to its usefulness as a research tool, the virtual reef can be used for educational purposes or simply for entertainment.

A scene from the virtual reef featuring both the fish and coral models is shown in Fig. 7.

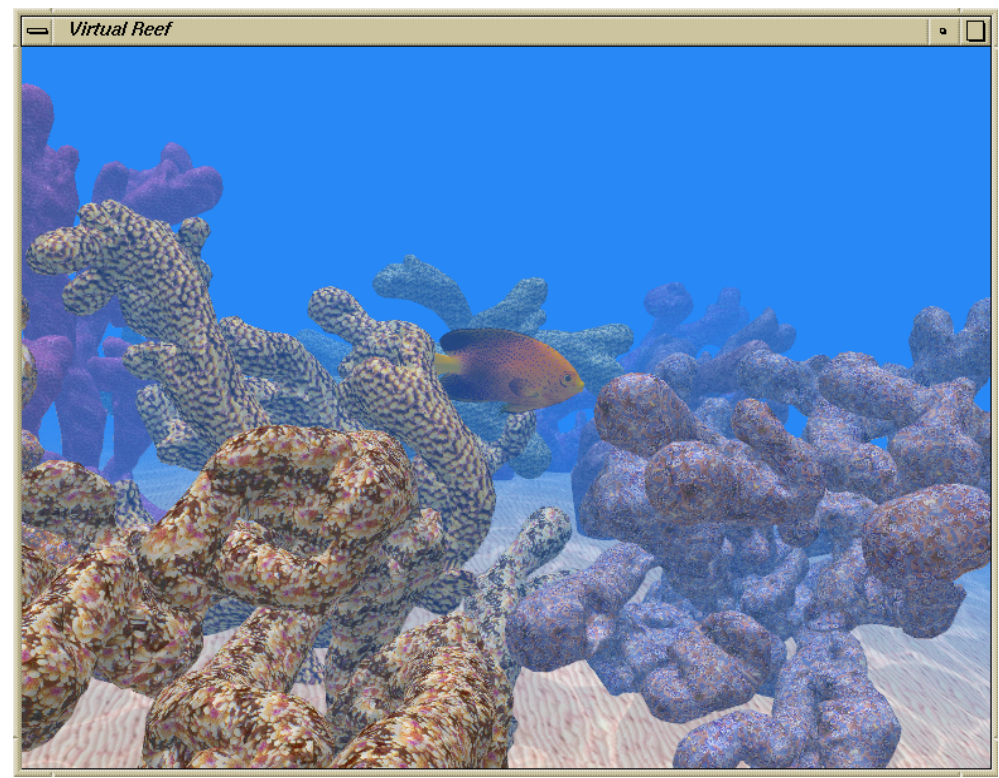

Fig. 7. Underwater scene taken from the Virtual Reef

The virtual reef is currently used for displaying dynamic datasets from both real-time and non-real-time simulation. Further development will focus on the display of observational datasets and the construction of a hydrodynamic model to simulate the flow of nutrients and its effect on fish movement.

\section{Acknowledgements.}

This work was carried out in the Visualisation and Advanced Computation Laboratory at the University of Queensland, using machines funded by the Queensland Parallel Supercomputing Foundation and the Australian Partnership for Advanced Computing. I would like to thank Dr Jaap Kaandorp for many useful discussions regarding the coral growth algorithm. 


\section{References}

1. Kaandorp, J.A., Kübler, J.E.: The algorithmic beauty of seaweeds, sponges, and corals. Springer-Verlag, Berlin Heidelberg New York (2001)

2. Kaandorp, J.A.: Fractal Modelling: Growth and Form in Biology. Springer-Verlag, Berlin Heidelberg New York (1994)

3. Kaandorp, J.A., Sloot, P.M.A.: Parallel simulation of accretive growth and form in three dimensions. BioSystems. 44 (1997) 181-192

4. Tu, X.: Artificial Animals for Computer Animation: Biomechanics, Locomotion, Perception and Behaviour. Lecture Notes in Computer Science, Vol. 1635. SpringerVerlag, Berlin Heidelberg New York (1999)

5. Press, W.H., Teukolsky, S.A., Vetterling, W.T., Flannery, B.P.: Numerical Recipes in C. The Art of Scientific Computing. Cambridge University Press, Cambridge (1992)

6. Webb, P.W.: Form and Function in Fish Swimming. Scientific American. 251 (1984) $58-68$

7. OpenGL Performer: Silicon Graphics, Inc. Mountain View, USA

8. Dorbie, A.: Peremptory Performer: Aqua demonstration. Internet site: www.dorbie.com 